\title{
Prevalence of chronic heart failure and asymptomatic left ventricular dysfunction in the general population: methods and preliminary results of the HF-Pomorskie Study
}

\author{
Aleksandra Puch-Walczak ${ }^{1}$, Piotr Hoffman², Maria Dudziak ${ }^{3}$, Katarzyna Kunicka ${ }^{4}$, Łukasz Wierucki ${ }^{1}$, \\ Ewa Puzio ${ }^{3}$, Krzysztof Kuziemski ${ }^{5}$, Magdalena Lipczyńska², Lucyna Konieczna ${ }^{6}$, Tomasz Bączek ${ }^{6}$, \\ Wojciech Drygas ${ }^{7,8}$, Tomasz Zdrojewski ${ }^{1}$ \\ 'Department of Preventive Medicine and Education, Medical University of Gdansk, Gdansk, Poland \\ ${ }^{2}$ Department of Congenital Heart Diseases, Institute of Cardiology, Warsaw, Poland \\ ${ }^{3}$ Department of Cardiac Diagnostics, Medical University of Gdansk, Gdansk, Poland \\ ${ }^{4}$ Department of Hypertension and Diabetology, Medical University of Gdansk, Gdansk, Poland \\ ${ }^{5}$ Department of Pulmonology, Medical University of Gdansk, Gdansk, Poland \\ ${ }^{6}$ Department of Pharmaceutical Chemistry, Medical University of Gdansk, Gdansk, Poland \\ 'Department of Social and Preventive Medicine, Medical University of Lodz, Lodz, Poland \\ ${ }^{8}$ Department of Epidemiology, Cardiovascular Disease Prevention, and Health Promotion, Institute of Cardiology, Warsaw, Poland
}

\section{INTRODUCTION}

The growing epidemic of chronic heart failure (CHF) has been highlighted by World Health Organization and European Union experts. There are no studies assessing the clinical epidemiology of $\mathrm{CHF}$ and asymptomatic left ventricular dysfunction (LVD) according to the European Society of Cardiology (ESC) guidelines in the general population in Poland, which is one of the countries representing Central and Eastern Europe - a region of high cardiovascular risk. Few available estimates indicate that heart failure (HF) is present in about $1 \%$ to $2 \%$ of adults in developed countries [1].

The present study aimed to assess the prevalence of $\mathrm{CHF}$ and LVD in the northern region of Poland, i.e. Pomeranian Voivodeship. According to 2014 Central Statistical Office data, the number of residents in this region is 2,302,077, including $1,855,474$ adults (mean age 39.8 years), which is similar to the mean age of the general Polish population [2].

\section{METHODS}

The study was conducted in 2014-2016 in a representative sample of Pomeranian Voivodeship residents aged 20 years or older. Sample selection was based on a three-stage approach. First, two rural communities (less than 8000 residents), two town communities (8000 to 40,000 residents), two middle-sized city communities (more than 40,000 residents), and one large-city community (more than 200,000 residents) were chosen by drawing lots, followed by drawing streets in urban communities, and finally drawing 100 individuals in each community proportionally to age and sex structure [3]. Study participants were randomly selected using the personal identification number (PESEL). Invitation letters were sent to all randomly chosen participants.

Additionally, we recruited 30 consecutive ambulatory patients from a mixed urban-rural area (four women and 26 men) with confirmed diagnosis of CHF and New York Heart Association (NYHA) class II or III, who were previously hospitalised in a regional hospital.

\section{Study protocol}

The study included evaluation of cardiovascular risk factors and comprehensive diagnostic investigations for HF according to the ESC criteria: (1) questionnaire; (2) anthropometric measurements - body weight, height, waist circumference, hip circumference; (3) blood pressure measurements; (4) echocardiography, electrocardiogram (ECG), and spirometry testing; (5) blood laboratory tests: N-terminal pro-B-type

\section{Address for correspondence:}

Aleksandra Puch-Walczak, MD, Department of Preventive Medicine and Education, Medical University of Gdansk, ul. Dębinki 7, budynek nr 1, 80-211 Gdańsk Poland, tel/fax: +48 5834919 75, e-mail: apw@gumed.edu.pl

Received: 6.09.2018 Accepted: 26.09.2018 Available as AoP: 26.09.2018

Kardiologia Polska Copyright (c) Polish Cardiac Society 2018 
natriuretic peptide (NT-proBNP), creatinine, lipids, alanine aminotransferase, total bilirubin, high-sensitivity $\mathrm{C}$-reactive protein, apolipoprotein A1, and apolipoprotein B (analytical methods are presented in Appendix 1 - see journal website); (6) blood plasma and urine analysis to identify biomarkers of $\mathrm{CHF}$ and cardiovascular disease (Appendix 2 - see journal website).

First, a nurse visited the participant at home, where the participant was given information regarding the study protocol, aims, and rationale. Then, in subjects who signed an informed consent to participate in the study, medical history was taken. This was followed by anthropometric and blood pressure measurements. Next, the recruited individual was invited to a local study centre (in the same place of residence) to undergo echocardiography, spirometry, and ECG, according to a uniform and standardised protocol.

\section{Questionnaire}

The questionnaire included 28 closed questions on details of medical history: chronic diseases, medications taken, social history including tobacco and alcohol use, and family history. In addition, the questionnaire included closed questions regarding HF symptoms (dyspnoea, reduced exercise tolerance) to implement the NYHA classification, as recommended in the ESC guidelines [4].

\section{Blood pressure measurements}

During the first visit, blood pressure was measured three times on the right arm using an automated oscillometric monitor, A\&D UA-767 (A\&D Company, Tokyo, Japan). Measurements were performed in accordance with the ESC/European Society of Hypertension guidelines [5].

\section{Electrocardiogram and echocardiography}

Echocardiography was performed by experienced echocardiographers, using a protocol developed by a group of experts based on the European Association of Cardiovascular Imaging and American Society of Echocardiography guidelines [6, 7]. The Siemens Acuson S2000 system (Siemens, Erlangen, Germany) was applied in standard views: parasternal, apical (two- and four-chamber), substernal, and suprasternal, using two-dimensional, M-mode, and Doppler imaging. The geometry of the left ventricle, right ventricle, and both atria was evaluated, along with valve morphology and function. Ejection fraction (EF) was calculated using the Simpson method. Relaxation abnormalities and left ventricular filling pressures were assessed to evaluate diastolic dysfunction. Echocardiographic recordings were evaluated off-line in two academic centres, and the sampling range (evaluation of the same examination by two independent experts) was $35 \%$. A 12-lead ECG was recorded at rest using an ELI 280 device (Moratara, Milwaukee, WI, USA).

\section{Spirometry}

Respiratory function testing (static and dynamic spirometry) has a scientific and clinical value only if performed with the utmost care and commitment from both the patient and the operator. For the purpose of our study, a MasterScreen Pneumo spirometer (CareFusion, Höchberg, Germany) was used, and the test was performed in accordance with the American Thoracic Society/European Respiratory Society guidelines [8].

\section{Blood and urine tests (Appendix 1)}

Venous blood was collected to a lithium heparin vacuum tube from each participant. In addition, a morning urine sample was provided by each participant, using standard urine collection rules. Within 30 min of collection, blood was centrifuged for $10 \mathrm{~min}$ at $1200 \mathrm{rpm}$. The obtained samples were transferred to secondary microtubes and frozen at $-20^{\circ} \mathrm{C}$. The urine samples were also frozen at $-20^{\circ} \mathrm{C}$. Within two days, samples were transported on dry ice to a central laboratory, where NT-proBNP and other laboratory parameters were assayed using Dimension EXL 200 and Immulite 1000 analysers (Siemens, Erlangen, Germany).

In addition, the concentration of steroids and amino acids in urine and blood was determined to search for new biomarkers of HF (Appendix 2 - see journal website).

Statistical analyses were performed using the R software (version 3.2.3). More information about the analysis is presented in Appendix 3 (see journal website).

The study design was approved by the Ethics Committee of the Medical University of Gdansk (approval no. NKBBN/421/2013 of 17 September 2013).

\section{RESULTS}

Overall, 700 individuals were invited to participate in the study. Of those, 148 addresses were incorrect or the individual could not be contacted. Finally, 313 individuals (170 women, 143 men) aged 20 years or older (mean age $55.2 \pm 15.3$ ) agreed to take part in the study and were examined. The response rate among all eligible subjects was $56.7 \%$. It was higher in rural and town communities compared to city communities (75\% vs. $42.7 \%$ ). Selected locations with the response rate are shown in Table 1.

The reference $\mathrm{CHF}$ group included 30 consecutive patients (four women and 26 men) with CHF (NYHA class II or III) diagnosed in the regional hospital. The mean age in the reference group was $65 \pm 11.1$ years.

\section{DISCUSSION}

Our study aimed to evaluate the prevalence of CHF and LVD in the Pomeranian Voivodeship. To our knowledge, this is the first attempt in Poland to examine a representative general population sample, using all current diagnostic clinical criteria necessary to establish CHF. The diagnosis of HF according to 
Table 1. Sample size and response rates according to communities

\begin{tabular}{|lccc|} 
Community & $\begin{array}{c}\text { Population } \\
\text { size }\end{array}$ & $\begin{array}{c}\text { Number } \\
\text { of examined } \\
\text { patients }\end{array}$ & $\begin{array}{c}\text { Response } \\
\text { rate [\%] }\end{array}$ \\
\hline Rakowiec & 1328 & 54 & 75 \\
Kepice & 3737 & 51 & 72 \\
Czersk & 10,030 & 44 & 61 \\
Kartuzy & 14,840 & 48 & 58 \\
Tczew & 60,573 & 42 & 46 \\
Gdynia & 247,820 & 39 & 48 \\
Gdansk & 461,489 & 35 & 42.70 \\
All & & 313 & 56.70 \\
\hline
\end{tabular}

2012 [9] and 2016 [4] ESC guidelines requires the presence of symptoms and signs, including dyspnoea, reduced exercise tolerance, cough, or oedema, along with morphological or functional echocardiographic abnormalities. Thus, the diagnostic algorithm includes physical examination, detailed medical history, ECG, and echocardiography. In addition, NT-proBNP or B-type natriuretic peptide level measurement is recommended in all patients with suspected HF [4].

Our initial results indicate that the response rate differed between smaller and larger communities. It seems that in smaller communities, the diagnostic tests offered in this study, i.e. spirometry and echocardiography, were more attractive for potential participants. Both tests are commonly performed, but in the public health care system in Poland they may be ordered only by a specialist, and thus the waiting time is up to several weeks. The availability of these diagnostic tests in larger communities is better, and thus it seems that residents of rural and town communities were more willing to participate given the opportunity to undergo these tests.

In Poland, all previous estimates on $\mathrm{CHF}$ have been based using complex ESC clinical criteria only in patients presenting to hospitals or primary care physicians [10-12]. No study targeted a representative general population sample, except the PolSenior [13] and the NATPOL 2011 studies. However, these two nationwide surveys were only based on medical history and NT-proBNP measurements, and they did not include ECG and echocardiography. Among subjects above 60 years of age included in the PolSenior study, NT-proBNP level $>2000 \mathrm{pg} / \mathrm{mL}$, consistent with a probable diagnosis of HF based on the 2008 ESC guidelines [14], was identified in $6.1 \%$ of the subjects [13]. The protocol of the NATPOL 2011 study, performed in subjects aged 18-79 years, included questions regarding previous diagnosis of $\mathrm{CHF}$, along with plasma NT-proBNP level measurement. The prevalence of $\mathrm{CHF}$ based on the questionnaire was $4.3 \%$. In Western Europe and the United States, only regional studies were performed. More details about the EPICA Study, the Rotterdam Heart Study, and the Olmsted County Study are presented in Appendix 4 (see journal website).

\section{Conflict of interest: none declared}

\section{References}

1. Mosterd A, Hoes AW. Clinical epidemiology of heart failure. Heart. 2007; 93(9): 1137-1146, doi: 10.1136/hrt.2003.025270, indexed in Pubmed: 17699180.

2. Central Statistical Office, Branch Yearbooks, Demographic Year-book of Poland, Warsaw. 2012.

3. Drygas W, Niklas AA, Piwońska A, et al. Multi-centre National Population Health Examination Survey (WOBASZ II study): assumptions, methods, and implementation. Kardiol Pol. 2016; 74(7): 681-690, doi: 10.5603/KP.a2015.0235, indexed in Pubmed: 26620680.

4. Ponikowski P, Voors A, Anker S, et al. 2016 ESC Guidelines for the diagnosis and treatment of acute and chronic heart failure. Eur J Heart Failure. 2016; 18: 891-975.

5. Mancia G, De Backer G, Dominiczak A, et al. 2007 Guidelines for the management of arterial hypertension: the task force for the management of arterial hypertension of the European Society of Hypertension (ESH) and of the European Society of Cardiology (ESC). Eur Heart J. 2007; 28: 1462-1536.

6. Garbi M, Edvardsen T, Bax J, et al. EACVI appropriateness criteria for the use of cardiovascular imaging in heart failure derived from European National Imaging Societies voting. Eur Heart J Cardiovasc Imaging. 2016; 17(7): 711-721, doi: 10.1093/ehjci/jew081, indexed in Pubmed: 27129538.

7. Nagueh S, Smiseth O, Appleton C, et al. Recommendations for the evaluation of left ventricular diastolic function by echocardiography: an update from the American Society of Echocardiography and the European Association of Cardiovascular Imaging. J Am Soc Echocardiogr. 2016; 29(4): 277-314, doi: 10.1016/j. echo.2016.01.011.

8. Miller MR, Hankinson J, Brusasco V, et al. ATS/ERS Task Force. Standardisation of spirometry. Eur Respir J. 2005; 26(2): 319-338, doi: 10.1183/09031936.05.00034805, indexed in Pubmed: 16055882

9. McMurray J, Adamopoulos S, Anker S, et al. ESC Guidelines for the diagnosis and treatment of acute and chronic heart failure 2012, Eur J Heart Failure. 2012; 14: 803-869.

10. Rywik TM, Kołodziej P, Targoński R, et al. Characteristics of the heart failure population in Poland: ZOPAN, a multicentre national programme. Kardiol Pol. 2011; 69(1): 24-31, indexed in Pubmed: 21267960.

11. Rywik TM, Zieliński T, Piotrowski W, et al. Heart failure patients from hospital settings in Poland: population characteristics and treatment patterns, a multicenter retrospective study. Cardiol J. 2008; 15(2): 169-180, indexed in Pubmed: 18651402.

12. Lipczyńska M, Szymański P, Klisiewicz A, et al. Detection of heart failure at a primary care practice in a small town in Central Poland. Kardiol Pol. 2012; 70(10): 1003-1008, indexed in Pubmed: 23080089.

13. Nadrowski P, Chudek J, Grodzicki T, et al. Plasma level of $\mathrm{N}$-terminal pro brain natriuretic peptide (NT-proBNP) in elderly population in Poland - the PolSenior Study. Exp Gerontol. 2013; 48(9): 852-857, doi: 10.1016/j.exger.2013.05.060, indexed in Pubmed: 23770107.

14. Dickstein K, Cohen-Solal, Filippatos C. et al. 2008 ESC Guidelines for the diagnosis and treatment of acute and chronic heart failure. Eur Heart J. 2008; 29: 2388-2442.

Cite this article as: Puch-Walczak A, Hoffman P, Dudziak M, et al. Prevalence of chronic heart failure and asymptomatic left ventricular dysfunction in the general population: methods and preliminary results of the HF-Pomorskie Study. Kardiol Pol. 2018; 76(11): 1567-1569, doi: 10.5603/KP.a2018.0201. 